\title{
Efeito de Diferentes Intervalos de Recuperação em um Programa de Treinamento de Força para Indivíduos Treinados
}

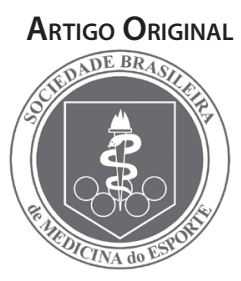

\author{
Effects of Different Rest Intervals in a Resistance Training Program for \\ Trained Individuals
}

Roberto Simão

Marcos Polito ${ }^{2}$

Walace Monteiro $3,4,5$

\section{Escola de Educação Física e Desporto - Universidade Federal do Rio de Janeiro (EEFD/UFRJ). \\ 2. Universidade Gama Filho (CEPAC) \\ 3. Programa de Pós-Graduação em Ciências da Atividade Física \\ - Universidade Salgado de Oliveira (UNIVERSO). \\ 4. Laboratório de Fisiologia do Exercício - Instituto de Ciências da Atividade Física da Aeronáutica (ICAF). \\ 5. Laboratório de Atividade Física e Promoção da Saúde - Universidade do Estado do Rio de Janeiro (LABSAU-UERJ).}

\section{Endereço para correspondência:} Walace Monteiro

Laboratório de Atividade Física e Promoção da Saúde. Universidade do Estado do Rio de Janeiro. Rua São Francisco Xavier, 524 $8^{0}$ and., sala 8.133, Bloco F. 20599-900 - Rio de Janeiro, RJ. E-mail:wdm@uerj.br

Submetido em 13/03/2006 Versão final recebida em 18/07/2006 Aceito em 20/12/2006

\begin{abstract}
RESUMO
São escassos na literatura estudos que investigaram a influência de diferentes intervalos de recuperação em um treinamento nos exercícios resistidos (ER) aplicado em longo prazo. O objetivo do presente estudo foi verificar a influência de dois diferentes intervalos de recuperação entre séries para grupos musculares distintos, durante oito semanas de treinamento. Preencheram os requisitos de inclusão no estudo 12 homens, sendo seis em cada grupo (26,4 $\pm 5,1$ anos; $181,3 \pm 6,2 \mathrm{~cm} ; 85,9 \pm 7,6 \mathrm{~kg})$. Os testes de carga em 10RM foram coletados em dois dias distintos e a aplicação no teste 10RM obedeceu à seguinte ordem: supino horizontal (SH), leg-press $45^{\circ}(\mathrm{LP})$ e rosca bíceps (RB). O primeiro grupo treinou com 1 min de intervalo, enquanto o segundo com 3 min de intervalo entre séries. O treinamento compreendeu três sessões semanais, realizadas em dias alternados (24 sessões). Realizaram-se três séries de cada exercício com os respectivos intervalos e, somente após as três séries no mesmo exercício, executava-se a seqüência no exercício posterior. A ordem do treinamento foi SH, LP, RB, hack machine, puxada pela frente no pulley alto, abdominal flexão parcial e tríceps no pulley. Para acompanhar o efeito dos diferentes intervalos nas três situações de medida, foi realizada uma ANOVA de uma entrada com medidas repetidas, seguida de verificação post-hoc de Tukey $(p<0,05)$. Em nenhuma das avaliações realizadas, observou-se diferença significativa entre as cargas para 10RM, quando comparados os intervalos de recuperação entre si. Concluiu-se que, independentemente do intervalo de recuperação adotado, não houve diferenças significativas nas cargas obtidas para 10RM nos ER conduzido por oito semanas. Para esclarecer a influência dos intervalos no desempenho da força, sugere-se a realização de estudos futuros com maiores tempos de acompanhamento e amostras com níveis de aptidão diferenciada.
\end{abstract}

Palavras-chave: exercícios resistidos, força, intervalos entre séries, treinamento, recuperação.

\begin{abstract}
It is scanty in the literature studies who investigated chronic effects in different rest intervals in resistance exercise (RE). The aim of the present study was to verify the influence of two different rest intervals between sets to distinct muscular groups, over eight weeks on RE training. Participated 12 men in this study, being six in each group (26.4 \pm 5.1 years; $181.3 \pm 6.2 \mathrm{~cm} ; 85.9 \pm 7.6 \mathrm{~kg}$ ). The 10RM test was made in two days (test and re-test) and was in the following order: bench press (BP), leg-press $45^{\circ}(\mathrm{LP})$ and biceps curl (BC). The first group trained with 1 min rest interval, and the other one with 3 min rest interval between sets. The total training consisted in three sessions per week, realized in alterned days (24 sessions). The subjects did three sets of each exercise in the respective intervals, and only after three sets in the same exercise, they go on in the next exercise. The exercise order was: BP, LP, BC, hack machine, lat pull down, abdominals and triceps pull down. A one-way ANOVA was used and a Tukey post hoc test was performed where indicated $(p<0.05)$ to verify the interval rest effects on the three measures. No significant differences between the 10RM loads were observed when compared the different rest intervals. We conclude that independent of rest interval, no significant differences were observed in 10RM loads in RE during eight weeks. To clarify the rest intervals influence on strength training, we suggest new future studies with samples in different levels of physical fitness and training in longer durations.
\end{abstract}

Keywords: resistance training, strength, rest intervals between sets, training, recovery.

\section{INTRODUÇÃO}

Os exercícios resistidos (ER) são eficientes para aumentar força, hipertrofia, potência e resistência muscular, mas, dependendo dos objetivos e das diferenças individuais, os padrões de prescrição podem variar bastante ${ }^{(1)}$. Uma série de variáveis devem ser controladas na prescrição dos ER, dentre as quais podemos destacar a ordem dos exercícios, o intervalo entre as séries e sessões, a freqüência semanal, o núme- ro de repetições e séries e a intensidade das cargas trabalhadas ${ }^{(2-5)}$.

Dentre as variáveis citadas uma que ainda vem sendo pouco estudada é a duração dos períodos de intervalo entre as séries e exercícios, exercendo influência direta na determinação do estresse do treino e no total da carga que pode ser manipulada ${ }^{(3)}$. Essa variável é considerada de grande importância ao se elaborar um programa de ER, e diferentes períodos de intervalo entre as séries e exercícios tem sido adotados em função dos 
objetivos a serem alcançados ${ }^{(4)}$. Alguns estudos de caráter longitudinal demonstraram maiores ganhos de força quando intervalos mais longos são aplicados em relação aos mais curtos (2-3 min vs. 30-40 segundos) ${ }^{(6-7)}$. No entanto, Ahtiainen et al. ${ }^{(8)}$ em recente investigação, verificaram que diferentes tempos de intervalo entre as séries ( 2 vs. 5 min), não exerceram diferenças significativas sobre os ganhos de força e hipertrofia durante dois protocolos de treinamento aplicados por seis meses.

Segundo Fleck e Kraemer ${ }^{(3)}$, a manipulação cuidadosa dos períodos de intervalo é essencial para evitar tensão inadequada e desnecessária no indivíduo durante o treinamento. Nesse contexto, além dos objetivos da prescrição, é provável que aspectos como os distintos grupos musculares, a ordenação dos exercícios, os graus de aptidão física dos praticantes e os sistemas de treinamento adotados possam influenciar na melhor definição dos intervalos entre séries e exercícios.

Até o presente momento, são escassos na literatura estudos que investigaram a influência de diferentes intervalos de recuperação em um treinamento aplicado em longo prazo ${ }^{(4-5)}$. Pode-se afirmar que as diferentes manipulações das variáveis de prescrição nos ER interferem na recuperação entre as séries ${ }^{(4)}$ e, conseqüentemente, nos efeitos fisiológicos do treinamento. Isso gera a possibilidade de realizar vários estudos com esse objetivo, principalmente com amostra constituída por sujeitos treinados. Assim, o presente estudo tem como propósito verificar a influência de dois diferentes intervalos de recuperação entre séries, aplicados em três exercícios para grupos musculares distintos, durante oito semanas em indivíduos treinados.

\section{MÉTODOS}

\section{Amostra}

Ingressaram no experimento 16 homens adultos, divididos em dois grupos. Com o decorrer do período de treinamento, preencheram os requisitos de inclusão no estudo 12 homens, sendo seis em cada grupo (26,4 \pm 5,1 anos; $181,3 \pm 6,2 \mathrm{~cm} ; 85,9 \pm 7,6 \mathrm{~kg}$ ), que não apresentavam contra-indicações para aplicação do teste de 10 repetições máximas (10RM). Todos os indivíduos eram fisicamente ativos, praticantes de ER havia pelo menos quatro anos, com freqüência mínima de três vezes semanais e que obtiveram presença em pelo menos $85 \%$ das sessões. Além da presença mínima, foi estabelecida como critério de inclusão a ausência de não mais do que uma vez por semana durante o período de treinamento. Foram excluídos do experimento indivíduos com PARQ positivo. Antes da coleta de dados, todos os indivíduos assinaram um termo de consentimento pós-informado, conforme a resolução do Conselho Nacional de Saúde (196/96).

\section{Teste de 10RM}

Os dados foram coletados em dois dias distintos. Na primeira visita, os indivíduos, após realizar as medidas antropométricas, executaram o teste de $10 \mathrm{RM}^{(9)}$. Após a obtenção das cargas máximas no teste de 10RM, os indivíduos descansaram por 48 horas e foram reavaliados para obtenção da reprodutibilidade das cargas no teste de 10RM (segundo dia de teste). Para tanto, considerou-se como 10RM a maior carga estabelecida em ambos os dias, com diferença menor que 5\%. Nos intervalos entre as sessões de testes não foi permitida a realização de exercícios, visando não interferir nos resultados obtidos.

A aplicação no teste 10RM obedeceu à seguinte ordem: supino horizontal (SH), leg-press $45^{\circ}(\mathrm{LP})$ e rosca bíceps com a barra livre (RB). Os exercícios foram selecionados devido à sua disseminação em centros de treinamento e facilidade de execução. Além disso, optou-se por selecionar exercícios que envolviam diferentes grupamentos musculares, o que permitiu avaliar a influência dos distintos intervalos de recuperação nesses grupamentos. Optou-se por aplicar o teste de 10RM em três exercícios para, posteriormente ao treinamento, compararmos os ganhos de força nesses exercícios.
Visando reduzir a margem de erro nos testes de 10RM, foram adotadas as seguintes estratégias ${ }^{(10)}$ : a) instruções padronizadas foram fornecidas antes do teste, de modo que o avaliado estivesse ciente de toda a rotina que envolvia a coleta de dados; b) o avaliado foi instruído sobre a técnica de execução do exercício; c) o avaliador estava atento quanto à posição adotada pelo praticante no momento da medida, pois pequenas variações no posicionamento das articulações envolvidas no movimento poderiam acionar outros músculos, levando a interpretações errôneas dos escores obtidos; d) estímulos verbais foram realizados a fim de manter alto o nível de estimulação; e) os pesos adicionais utilizados no estudo foram previamente aferidos em balança de precisão. Os intervalos entre as tentativas em cada exercício durante o teste de 10RM foram fixados entre 2 e 5 min ${ }^{(9)}$. Após obtenção da carga em um determinado exercício, intervalos não inferiores há 10 min foram dados, antes de passar-se ao teste no exercício seguinte. Após quatro e oito semanas de treinamento, a utilização do teste e reteste em $10 R M$ como no pré-treinamento foi adotada com objetivo de verificação da reprodutibilidade das cargas.

\section{PROTOCOLO DE TREINAMENTO}

Anteriormente à realização nos testes de 10RM (pré-treinamento), os sujeitos foram divididos em dois grupos. O primeiro grupo treinou com 1 min de intervalo, enquanto o segundo com 3 min de intervalo entre as séries. Ambos os grupos passaram por uma semana de familiarização aos exercícios (três sessões). Tal procedimento foi adotado para que todos os avaliados ficassem familiarizados com a seqüência de exercícios, cargas e intervalos. Em ambos os grupos realizou-se o sistema alternado por segmento. Após o período de familiarização, aplicaram-se os testes de 10RM, que foram repetidos ao final da quarta e oitava semana de treino. Todas as sessões foram acompanhadas sob a supervisão de um professor de educação física experiente na prescrição dos ER.

$O$ treinamento compreendeu três sessões semanais, realizadas em dias alternados, totalizando 24 sessões. A adesão ao programa de treinamento foi de 100\% para 10 integrantes da amostra e 91,6\% para dois integrantes. $\mathrm{O}$ treinamento no método alternado por articulação foi composto por sete exercícios realizados em três séries, variando entre oito a 10RM, com exceção do exercício abdominal, no qual foram executadas três séries de 15-20RM. Realizaram-se três séries de cada exercício com os respectivos intervalos e, somente após as três séries no mesmo exercício, executava-se a seqüência no exercício posterior com o mesmo intervalo. Para evitar fadiga demasiada, já que os indivíduos treinavam no limiar, alternaram-se os grupamentos musculares na seqüência de treinamento. Quando os indivíduos ultrapassavam ou ficavam aquém das faixas de repetições estabelecidas, as cargas eram reajustadas.

A ordem estabelecida para o treinamento foi a seguinte: supino horizontal, leg-press 45, rosca bíceps, hack machine, puxada pela frente no pulley alto, abdominal flexão parcial e tríceps no pulley. Antes de cada sessão de treinamento os sujeitos realizaram duas séries de aquecimento específico, envolvendo 15 repetições com 50\% da carga utilizada no primeiro e segundo exercício da seqüência. O intervalo adotado nos respectivos grupos foi similar entre as séries e exercícios.

\section{TRATAMENTO ESTATÍSTICO}

A determinação da reprodutibilidade nas cargas no pré-treinamento, quatro e oito semanas pós-treinamento em 10RM foi realizada através do coeficiente de correlação intraclasse, adicionada ao teste $t$ de Student pareado. Para acompanhar o efeito dos diferentes intervalos nas três situações de medida de 10RM, foi realizada uma ANOVA de uma entrada com medidas repetidas, seguida de verificação post-hoc de Tukey. Considerou-se como nível de significância $p<0,05$. Os dados foram analisados no programa Statistica 5.5 (Statsoft, EUA). 


\section{RESULTADOS}

Inicialmente, são ilustrados os resultados referentes à reprodutibilidade das cargas obtidas nos testes de 10RM nas diferentes situações de treinamento investigadas. Foram verificados elevados valores de coeficientes de correlação intraclasse nos exercícios no pré-treinamento: $\mathrm{SH} r=0,94$; $\mathrm{LPr}=0,92$ e RB $r=0,96$; quatro semanas de treinamento: $\mathrm{SH} r=0,96$; $\mathrm{LP} r=0,94$ e RB $r=0,96$; e oito semanas de treinamento: $\mathrm{SH} r=0,96 ; \mathrm{LP} r=0,94$ e RB $r=0,96$. Em adição, o teste $t$ de Student pareado não demonstrou diferença significativa entre as cargas obtidas para 10RM em cada exercício ( $p<0,05)$, tanto pré como quatro e oito semanas pós-treinamento. Os valores referentes ao pareamento das cargas nos três exercícios na situação de pré-treinamento foram: $p=0,154(\mathrm{SH}) ; p=0,057(\mathrm{RB}) ; p=0,136(\mathrm{LP})$, revelando que os dois grupos iniciaram o experimento com níveis de força homogêneos nos exercícios.

As figuras 1, 2 e 3 ilustram o comportamento das cargas em 10RM em relação aos intervalos de recuperação aplicados para cada um dos exercícios. No SH (figura 1), somente foi observada diferença significativa para os valores de base na oitava semana de treinamento, em ambos os intervalos de recuperação. Já nos exercícios RB (figura 2) e LP (figura 3), diferenças significativas em relação aos valores iniciais foram identificadas na quarta e na oitava semana de treinamento, também nos dois intervalos de recuperação. Em nenhum momento foi observada diferença significativa entre os intervalos de recuperação.

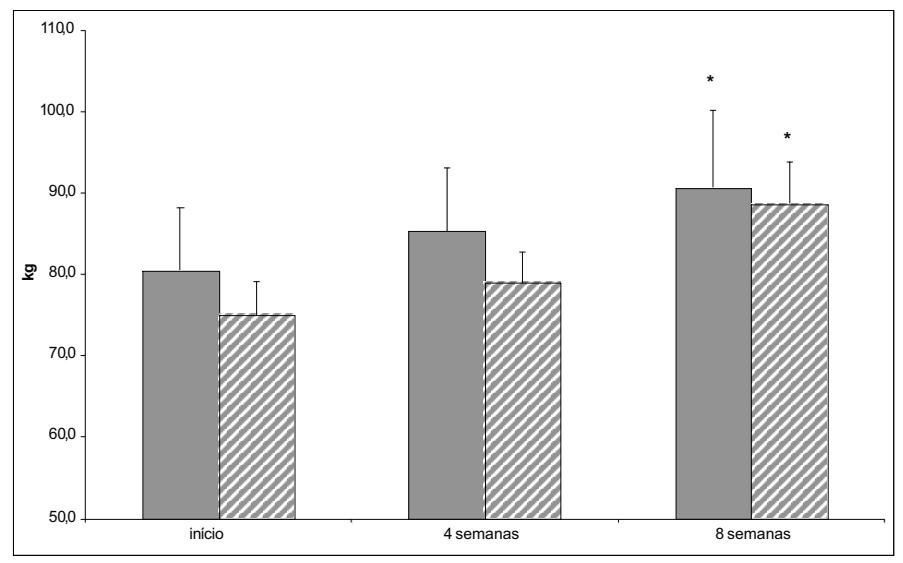

Figura 1. Comportamento da carga do exercício SH após oito semanas de treinamento com 1 min (coluna cinza) e 3 min (coluna listrada) de recuperação.

* diferença significativa $(p<0,05)$ em relação aos valores iniciais (pré)

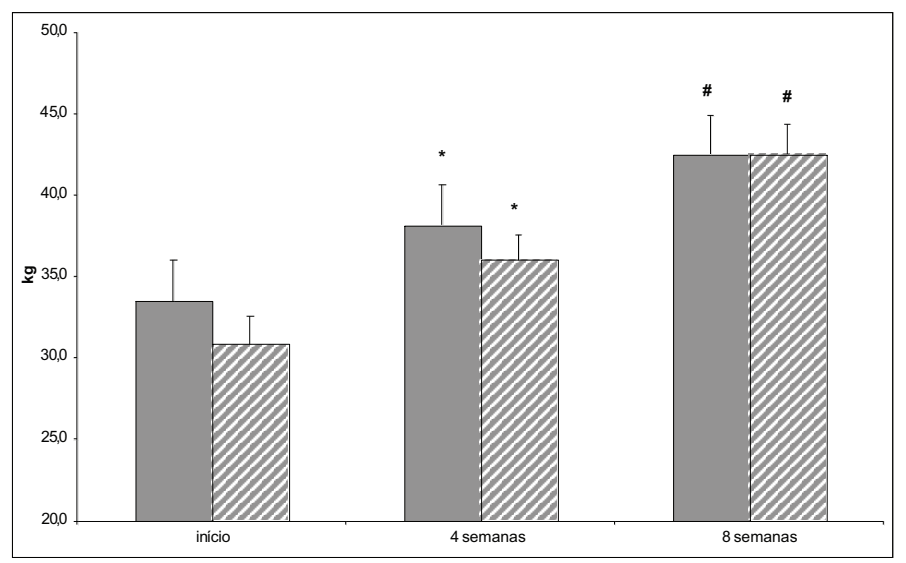

Figura 2. Comportamento da carga no exercício RB após oito semanas de treinamento com 1 min (coluna cinza) e 3 min (coluna listrada) de recuperação.

* diferença significativa $(p<0,05)$ em relação aos valores iniciais (pré)

\# diferença significativa $(p<0,01)$ em relação aos valores iniciais (pré)

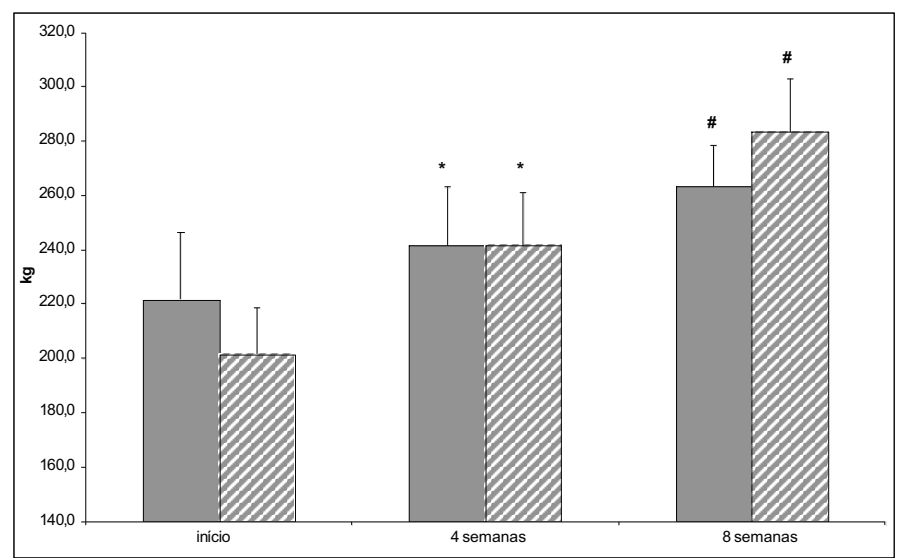

Figura 3. Comportamento da carga do exercício LP após oito semanas de treinamento com 1 min (coluna cinza) e 3 min (coluna listrada) de recuperação.

* diferença significativa $(p<0,05)$ em relação aos valores iniciais (pré)

\# diferença significativa $(p<0,01)$ em relação aos valores iniciais (pré)

\section{DISCUSSÃO}

De acordo com o posicionamento do American College of Sports Medicine $^{(1)}$ no treinamento de força para praticantes intermediários e avançados, devem ser utilizados intervalos entre 2 a 3 min para exercícios multi-articulares que envolvam massas musculares relativamente grandes. Já para exercícios uniarticulares, com menores massas musculares, recomenda-se um intervalo com um período mais curto, variando entre 1 e 2 min. O posicionamento destaca que essas faixas de intervalos parecem ser suficientes para provocar adequada recuperação entre as séries. Contudo, essa ainda é uma questão pouco explorada na literatura e as evidências sobre o assunto baseiam-se em alguns estudos relacionados aos efeitos agudos do treinamento.

Segundo Bird et al. ${ }^{(5)}$ ao prescrever os intervalos de recuperação em programas de ER para o desenvolvimento da potência muscular, são necessários de 5 a 8 min. No caso da força máxima, uma faixa de 3 a 5 min deve ser requerida. Já para o desenvolvimento da hipertrofia muscular, períodos de recuperação entre 1 e 2 min são habitualmente prescritos. No presente estudo, para obtenção das cargas em teste de 10RM, intervalos de 3 min não demonstraram ser estatisticamente diferentes, em relação aos intervalos de 1 min em todos os exercícios selecionados. Nesse caso, 1 e 3 min de descanso entre as séries e exercícios foram similares para os ganhos de força obtidos ao final de oito semanas de treinamento. Dessa forma, nossos resultados contrapõemse às recomendações apresentadas na literatura ${ }^{(1,5)}$. Cabe ressaltar que em ambas as referências citadas anteriormente, não foi feita nenhuma menção quanto ao tempo de acompanhamento do treinamento. Dessa forma, é possível que o tempo de intervalo utilizado para obtenção de melhores respostas agudas possa se diferenciar daqueles necessários mediante a exposição crônica ao treinamento.

Os resultados do presente estudo evidenciaram que em todos os exercícios e intervalos de recuperação investigados, bem como em nenhum dos momentos de avaliação, observou-se diferença significativa nos ganhos de força no teste de 10RM. Ao analisar os ganhos de força em cada intervalo estudado isoladamente, somente o exercício SH não apresentou significância nos ganhos de força entre a primeira e quarta semana. Já ao comparar a primeira e oitava semana de treinamento, todos os exercícios demonstraram ganhos de força de forma significativa no teste de 10RM.

Os mecanismos fisiológicos que tentam elucidar o papel da fadiga no desempenho nas séries e seus respectivos intervalos ainda são incipientes. Normalmente, fisiculturistas utilizam intervalos de recuperação cur- 
tos, causando elevado estresse muscular, devido principalmente a maior liberação dos hormônios anabólicos. Em adição, períodos de intervalos curtos são acompanhados de considerável desconforto muscular, devido à oclusão do fluxo sangüíneo, produção de lactato(11) e, conseqüentemente, diminuição da produção de força ${ }^{(12)}$. Entretanto, os levantadores de peso utilizam altas cargas de treinamento, mas com períodos de descanso elevados, quando comparados com os fisiculturistas. Alguns autores especulam que esse longo intervalo seria necessário para promover restabelecimento das funções orgânicas ${ }^{(3-5)}$, entre as quais podem ser destacadas as recuperações dos sistemas neural e energético.

Normalmente, são empregados intervalos de recuperação de 30 a 60 segundos para fisiculturistas e de 2 a 5 min para levantadores de peso $^{(3)}$. Isso porque intervalos iguais ou inferiores a 1 min limitam a recuperação das reservas de CP e ATP. Estima-se que a recuperação total de ATP dura, em média, de 3 a 5 min após exercício extenuante, enquanto a CP para recuperação total, em média, 8 min ${ }^{(13)}$. Outro fator importante que pode influenciar na recuperação entre as séries é o aumento nos níveis de lactato durante o treinamento de força intenso ${ }^{(3,14)}$. O tempo necessário para diminuição do lactato após os ER desempenhados em alta intensidade deve ser entre 4 e 10 min; tempos inferiores à faixa citada acarretam elevada concentração de íons de hidrogênio $\left(\mathrm{H}^{+}\right)$, diminuindo o $\mathrm{pH}$ intracelular, resultando em fadiga muscular ${ }^{(15-16)}$.

As evidências atuais baseiam-se em estudos de efeito agudo(12-13,17) não podendo ser extrapoladas para os que tratam dos efeitos crônicos, caso da presente investigação, cujos resultados não corroboram as evidências referentes aos efeitos agudos. Talvez essa diferença envolva um somatório de aspectos como as diferenças nas características das amostras e suas influências nas respostas fisiológicas obtidas em longo prazo. Em adição, as diferentes metodologias de treinamento podem afetar o comportamento de variáveis neuromusculares e hormonais decorrentes do treinamento.

Poucos experimentos analisaram a influência dos intervalos entre as séries nos ER em longo prazo e as diferenças metodológicas entre esses experimentos apresentam resultados que apontam para caminhos diferentes. Recentemente, Ahtiainen et al. ${ }^{(8)}$, verificando a influência de intervalos de 2 e 5 min nos ER com duração de seis meses, não observaram diferenças significativas nas adaptações hormonais e neuromusculares em homens treinados. Em contrapartida, Pincivero et al. ${ }^{(6)}$, após quatro semanas de treinamento isocinético, comparando 40 e 160 segundos de intervalo, verificaram que a força muscular dos posteriores de coxa respondem melhor a maior intervalo entre as séries do que a musculatura do quadríceps. Nesse caso, grupos musculares de tamanhos distintos apresentaram recuperação diferenciada. Cabe ressaltar que, nesse estudo, a amostra também foi composta por homens treinados. Já Robinson et al. ${ }^{(7)}$, verificando a influência de cinco semanas de treinamento em homens treinados, em três intervalos (180, 90 e 30 segundos) no agachamento concluíram que, quanto maior o intervalo entre as séries, maiores serão os ganhos de força em termos percentuais no teste de 1RM.

Os dois estudos supracitados ${ }^{(6-7)}$ demonstram que maior período de intervalo promoveu maiores ganhos na força. Os estudos de Pincivero et al.(6) e Robinson et al. ${ }^{(7)}$ tiveram durações de quatro e cinco semanas, respectivamente. Não se sabe até que ponto a semelhança na duração total do treinamento pode ter influenciado na similaridade dos resultados entre os estudos. Em contraposição, Ahtiainen et al. ${ }^{(8)}$ comparando 2 e 5 min entre as séries, não verificaram alterações hormonais e neuromusculares significativas em seis meses de treinamento. Em nosso estudo, o treinamento foi conduzido por oito semanas com 24 sessões e freqüência semanal de três vezes. Mesmo não podendo afirmar que maior tempo de intervalo promoveu maiores ganhos de força, é importante destacar que, em termos percentuais, 3 min de intervalo foram superiores a 1 min, na evolução das cargas para 10RM. Não se sabe até que ponto a condução de um programa com as mesmas características com maior duração exerceria efeitos diferentes nos ganhos de força, provocando alterações significativas nos valores absolutos para 10RM, fato não observado em nosso experimento. Outro aspecto a ser levantado, e que traduz uma limitação do estudo, diz respeito ao tamanho da amostra que compôs cada grupo no presente experimento. Para que inferências mais consistentes possam ser realizadas, estudos futuros devem considerar maior número de sujeitos.

Em função dos resultados obtidos, concluiu-se que, independentemente do intervalo de recuperação adotado, não houve diferenças significativas nas cargas obtidas para 10RM nos ER conduzido por oito semanas. Para esclarecer a influência dos intervalos no desempenho da força, sugere-se a realização de estudos futuros com maiores tempos de acompanhamento, envolvendo amostras mais abrangentes e homogêneas quanto ao grau de condicionamento físico inicial e composição corporal, bem como o controle das condições alimentares dos voluntários.

Todos os autores declararam não haver qualquer potencial conflito de interesses referente a este artigo.

\section{REFERÊNCIAS BIBLIOGRÁFICAS}

1. American College of Sports Medicine. Position Stand: Progression Models in Resistance Training for Healthy Adults. Med Sci Sports Exer 2002;34:364-80.

2. Simão R, Farinatti PTV, Polito MD, Maior AS, Fleck SJ. Influence of exercise order on the number of repetitions performed and perceived exertion during resistive exercises. J Strength Cond Res 2005;11:152-6.

3. Fleck SJ, Kraemer WJ. Designing resistance training programs. Champaign: Human Kinetics, 2004.

4. Kraemer WJ, Ratamess NA. Fundamentals of resistance training: Progression and exercise prescription. Med Sci Sports Exerc 2004;36:674-88

5. Bird SP, Tarpenning M, Marino FE. Designing resistance training programmes to enhance muscular fitness. Sports Med 2005;35:841-51

6. Pincivero DM, Lephart SM, Karunakara RG. Effects of rest interval on isokinetic strength and functional performance after short term high intensity training. British J Sports Med 1997;31:229-34.

7. Robinson JM, Stone MH, Johnson RL, Penland CM, Warren BJ, Lewis RD. Effects of different weight training exercise/rest intervals on strength, power, and high intensity exercise endurance. J Strength Cond Res 1995;9:216-21.

8. Ahtiainen JP, Pakarinen A, Alen M, Kraemer WJ, Häkkinen K. Short vs. long rest period between the sets in hypertrophic resistance training: Influence on muscle strength, size, and hormonal adaptations in trained men. J Strength Cond Res 2005;19:572-82

9. Bachle TR, Earle RW. Essentials of strength training and conditioning. Champaign: Human Kinetics, 2000.

10. Monteiro W, Simão R, Farinatti PTV. Manipulação na ordem dos exercícios e sua influência sobre o número de repetições e percepção subjetiva de esforço em mulheres treinadas. Rev Bras Med Esporte 2005;11:146-50.

11. Tesh P, Larson L. Muscle hypertrophy in bodybuilders. Europ J Apply Phys 1982;49:301-6.

12. Willardson JM, Burkett $L N$. A comparison of 3 different rest intervals on the exercise volume completed during a workout. J Strength Cond Res 2005;19:23-6.

13. Weir JP, Wagner LL, Housh $T J$. The effect of rest interval length on repeated maximal bench presses. J Strength Cond Res 1994;8:58-60.

14. Kraemer WJ, Noble BJ, Clark MJ, Culver BW. Physiologic responses to heavy-resistance exercise with very short rest periods. Intern I Sports Med 1987;8:247-52.

15. Hultman E, Sjoholm H. Biomechanical causes of fatigue. Champaign: Human Kinetics, 1986.

16. Jones NL. $\left(\mathrm{H}^{+}\right)$Control in exercise: concepts and controversies. Champaign: Human Kinetics, 1990.

17. Larson Jr GD, Potteiger JA. A comparison of three different rest intervals between multiple squat bouts. J Strength Cond Res 1997;11:115-8. 